\title{
Mean Field with Tensor Force and Shell Structure of Exotic Nuclei
}

\author{
Takaharu Otsuka, ${ }^{1,2,3}$ Toshiaki Matsuo, ${ }^{4}$ and Daisuke Abe ${ }^{1}$ \\ ${ }^{1}$ Department of Physics, University of Tokyo, Hongo, Bunkyo-ku, Tokyo, 113-0033, Japan \\ ${ }^{2}$ Center for Nuclear Study, University of Tokyo, Hongo, Bunkyo-ku, Tokyo, 113-0033, Japan \\ ${ }^{3}$ RIKEN, Hirosawa, Wako-shi, Saitama 351-0198, Japan \\ ${ }^{4}$ Software Division, Hitachi Ltd., Yokohama, Kanagawa, Japan \\ (Received 9 May 2006; published 16 October 2006)
}

\begin{abstract}
The tensor force is implemented into the mean-field model so that the evolution of nuclear shells can be described for exotic nuclei as well as stable ones. Besides the tensor-force part simulating the meson exchange, the model is an extension of the successful Gogny model. One of the major issues of rareisotope beam physics is a reduced spin-orbit splitting in neutron-rich exotic nuclei. It will be shown that the effect of the tensor force on this splitting is larger than or about equal to the one due to the neutron skin. We will present predictions for stable and exotic nuclei with comparisons to conventional results and experimental data.
\end{abstract}

DOI: 10.1103/PhysRevLett.97.162501

PACS numbers: 21.60. $-\mathrm{n}, 21.10 . \mathrm{Pc}, 21.30 . \mathrm{Fe}$

Like other many-body quantal systems, the mean field governs largely the structure of nuclei. Recently it has been shown, by using the shell model, that the tensor force has a specific, robust, and systematic effect on the single-particle energies of nuclei, even breaking or creating magic numbers in some cases [1]. It is then very interesting to explore what can be seen by including the tensor force into the mean-field calculations. Note that in the shell model, the single-particle wave functions are given $a$ priori and are fixed more or less.

The existing successful and systematic mean-field calculations are either of Skyrme-type [2,3] or of Gogny-type [4]. In the former, the interaction is of zero-range nature with derivative couplings, while the tensor terms have been suppressed, probably because the number of free parameters is made as small as possible [5]. In the latter, the interaction is of finite-range nature $[4,6,7]$, while there is no tensor term either.

In this Letter, we present a new mean-field model which includes the tensor force explicitly and keeps the merits of the existing models. In exotic nuclei far from stability with large asymmetric ratios between the proton number $(Z)$ and the neutron number $(N)$, the single-particle properties may show distinct characteristic changes from those seen in usual stable nuclei. In particular, in neutron-rich exotic nuclei, the neutron skin can be created [8], which may give rise to a diffuse surface resulting in a reduced spin-orbit splitting $[9,10]$. The tensor force changes the spin-orbit splitting in a systematic and different way [1]. It should be very important to investigate the neutron-skin effect and the tensor-force effect within a single framework.

We introduce a new Gogny-type mean-field model, called GT2. All the terms of the Gogny interaction are kept, while their strength parameters are readjusted. The Gogny interaction contains central interactions with Gaussian-function dependences on the relative distance of nucleons $[4,6,7]$. Two ranges of Gaussians are included with full four spin-isospin coupling terms, ending up with eight terms. The tensor term is expressed as [1],

$$
V_{T}=\left(\vec{\tau}_{1} \cdot \vec{\tau}_{2}\right)\left(\left[\vec{s}_{1} \vec{s}_{2}\right]^{(2)} \cdot Y^{(2)}\right) f_{G}(r),
$$

where $\vec{\tau}_{1,2}\left(\vec{s}_{1,2}\right)$ denotes the isospin (spin) of nucleons 1 and $2,[]^{(K)}$ means the coupling of two operators in the brackets to an angular momentum (or rank) $K$, and $Y$ denotes the spherical harmonics for the relative coordinate. Here, $f_{G}(r)$ is a function of the relative distance, $r$. Similarly to the central part of the interaction, we take a Gaussian for $f_{G}(r)$ too, for simplicity, with the range $1.2 \mathrm{fm}$, i.e., the longer range of the central part. The overall strength of $f_{G}(r)$ is then determined so that its volume integral reproduces that of the AV8' [11], resulting in $f_{G}(r=0)=3156(\mathrm{MeV})$. Since AV8' tensor potential has a Yukawa form, the GT2 tensor potential is stronger than AV8' in the inner part, but damps quickly, becoming smaller than AV8' for $r>2 \mathrm{fm}$. Note that the tensor part of AV8' potential is similar to that of M3Y potential [12]. In the future, the tensor potential must be more realistic, but the present simple form seems to be reasonable as a first study.

We shall discuss properties of the GT2 interaction in comparison to the D1S interaction [7], the most frequently used Gogny interaction. Although details of the central part of GT2 are not relevant to the major points of this Letter, main features considered to fit the parameters of the central part of GT2 are, (1) the symmetric nuclear matter shows the same minimum $E / A$ and its Fermi momentum as those of D1S, (2) the incompressibility is $K=228 \mathrm{MeV}$, (3) experimental binding energies of ${ }^{16} \mathrm{O},{ }^{40} \mathrm{Ca},{ }^{48} \mathrm{Ca},{ }^{56} \mathrm{Ni},{ }^{132} \mathrm{Sn}$, and ${ }^{208} \mathrm{~Pb}$ are reproduced with an accuracy of $\sim 1 \%$ from experiment, (4) the $\sigma \sigma \tau \tau$ central potential is tuned so that its overall strength behaves being positive [13]. The values of the parameters in the usual notation are, for the range 0.7 (1.2) fm, $W=2311$ (-339), $B=-3480$ (388), $H=2962$ $(-370), M=-2800(260) \mathrm{MeV}$. The signs are opposite 
from D1S interaction, because of the sign change in the $\sigma \sigma \tau \tau$ part. However, the triplet-even potential is very similar between D1S and GT2, and the singlet-even is also very close outside $1 \mathrm{fm}$.

The strength of 2-body spin-orbit (LS) force is $W_{0}=$ $160 \mathrm{MeV} \mathrm{fm}^{5}$, while the density-dependent force is given by $t_{3}=1400 \mathrm{MeVfm}^{4}$ and $x_{0}=1$ with the $\rho^{1 / 3}$ dependence.

While the nuclear radii are not used for the fit of GT2, they turn out to be almost the same as the corresponding values by D1S. The surface diffuseness does not differ much either between GT2 and D1S.

Regarding the pairing interaction, a new aspect of GT2 is the contribution from the tensor force. Including this, we should refine the central part of GT2, examining pairing properties in addition to single-particle properties. The present work is focused on Hartree-Fock (HF) calculation, and pairing issues are left open for Hartree-FockBogoliubov calculation to be done. Thus, there may be certain rooms for refinement of the parameters, but the GT2 interaction appears to be good enough for the present purpose, as we shall see.

We here recall the shell evolution due to the tensor force [1]. We consider orbits with orbital angular momenta $l$ and $l^{\prime}$, to be coupled with spin. For simplicity, protons are in either $j_{>}=l+1 / 2$ or $j_{<}=l-1 / 2$, while neutrons are in either $j_{>}^{\prime}=l^{\prime}+1 / 2$ or $j_{<}^{\prime}=l^{\prime}-1 / 2$. The monopole effect of the tensor force is (i) attractive between $j_{>}$and $j_{<}^{\prime}$ and between $j_{<}$and $j_{>}^{\prime}$, (ii) repulsive between $j_{>}$and $j_{>}^{\prime}$ and between $j_{<}$and $j_{<}^{\prime}$. Its magnitude depends on $l$ and $l^{\prime}$ and also on the radial overlap.

Figures 1(a) and 1(b) show neutron single-particle energies (SPE) for $1 d_{5 / 2,3 / 2}, 2 s_{1 / 2}$, and $1 f_{7 / 2}$ orbits of $N=20$ isotones as a function of $Z$. The calculated SPE's mean, in this Letter, spherical HF SPE's at (sub-)shell closures. The calculation is carried out on harmonic oscillator bases up to $13 \hbar \omega$, and the center-of-mass correction term and Coulomb term are included in the Hamiltonian matrix elements. The pairing interaction enters only through its monopole component. Some positive energy levels are shown in Fig. 1 for relevant orbits with large l's. As more protons occupy $1 d_{5 / 2}$, the neutron orbits are shifted down. Following the general rule mentioned above, the monopole effect of the tensor force between proton $d_{5 / 2}$ and neutron $d_{3 / 2}$ is attractive, whereas that between proton $d_{5 / 2}$ and neutron $f_{7 / 2}$ is repulsive. One sees these trends very clearly in Fig. 1(b): $d_{3 / 2}$ and $f_{7 / 2}$ repel each other in going from $Z=8$ to 14 , leaving a wide $N=16$ gap for $Z=8$ while the usual $N=20$ gap becomes wide for $Z=$ 14 . Thus, the magic gap changes depending on $Z$. The trends seen in Fig. 1(b) are consistent with recent experiments on exotic oxygen [14], neon [15], sodium [16,17], and magnesium [18] isotopes, suggesting narrow $N=20$ gap for smaller $Z$. Since D1S does not include the tensor, such trends do not show up, and the splitting between $d_{3 / 2}$
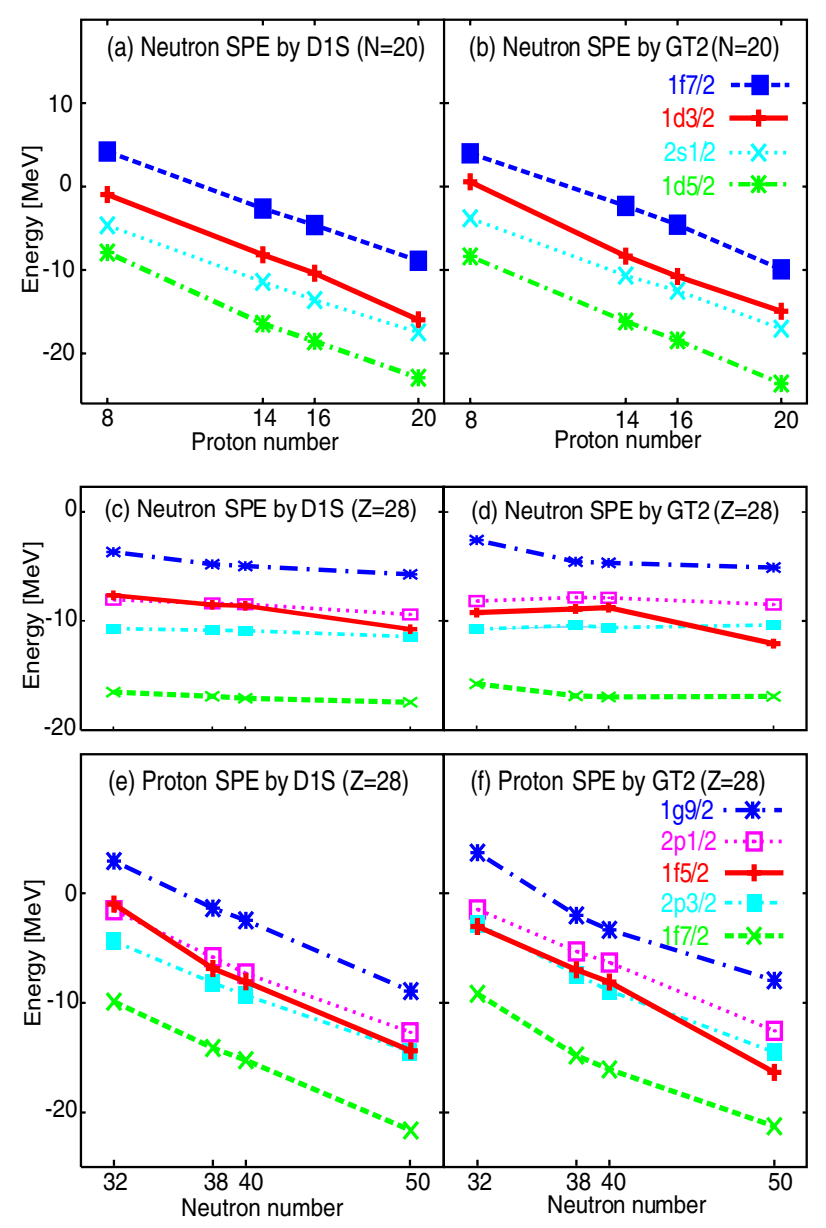

FIG. 1 (color online). SPE's calculated by D1S and GT2 interactions. (a),(b) SPE's of neutrons for $N=20$ as a function of $Z$. (c),(d) SPE's of neutrons for $Z=28$ as a function of $N$. (e),(f) SPE's of protons for $Z=28$ as a function of $N$.

and $d_{5 / 2}$ moves in the opposite way [See Fig. 1(a)] basically due to the opposite $\sigma \sigma \tau \tau$ central potential compared to GT2. We note that ${ }^{28} \mathrm{O}$ is unbound slightly in Fig. 1(b), while bound in Fig. 1(a). The neutron $d_{5 / 2}$ comes down almost as much as $d_{3 / 2}$ from $Z=8$ to 14 , because the $T=$ 1 attractive pairing contained in the central part of GT2 gives rise to an attractive monopole effect from protons in $d_{5 / 2}$ on a neutron in $d_{5 / 2}$.

Figures 1 (c)-1(f) show SPE's for $1 f_{7 / 2,5 / 2}$ and $2 p_{3 / 2,1 / 2}$ of $Z=28(\mathrm{Ni})$ isotopes as a function of $N$. As more neutrons occupy $1 g_{9 / 2}$, the neutron orbits stay rather constant in both D1S and GT2 calculations, except that $1 f_{7 / 2}$ and $1 f_{5 / 2}$ come close at $N=50$. In the $N=50$ isotope, the neutron skin [8] is created.

The spin-orbit splitting is generated, in the Skyrme and Gogny models, from the two-body LS interaction, and the resultant splitting of neutron and that of proton are calculated, respectively, from [3],

$$
g_{n}=2 \frac{\partial \rho_{n}}{\partial r}+\frac{\partial \rho_{p}}{\partial r} \quad \text { and } \quad g_{p}=\frac{\partial \rho_{n}}{\partial r}+2 \frac{\partial \rho_{p}}{\partial r},
$$


where $\rho_{p}\left(\rho_{n}\right)$ denotes the density distribution of protons (neutrons), and $r$ implies the distance from the center of the nucleus. The spin-orbit splitting is calculated by integrating $r^{2} R(r)^{2} g(r) / r$, where $R$ means the radial wave function of the relevant orbit. Figures 2(e) and 2(f) show $g_{p, n}(r)$, while Figs. 2(a)-2(d) present $r^{2} R(r)^{2}$. Clearly, $R(r)$ and $g(r)$ are peaked at about the same $r$. To go more into detail, however, $g_{p, n}$ in Fig. 2 move outwards as $N$ changes from 40 to 50 .

Since each $R(r)$ is basically unchanged between $N=40$ and 50 , the $1 f_{5 / 2}-1 f_{7 / 2}$ splitting is expected to be reduced by the growing mismatch between the peaks of $R(r)$ and $g(r)$ in going from $N=40$ to 50 . This is different from the conventional explanation [10] that in neutron-rich nuclei the density decreases more slowly at the surface as a function of $r$ and thereby the magnitude of $g(r)$ becomes smaller, giving rise to a reduced spin-orbit splitting. For clarification, this conventional picture will be referred to as scale-type (s-type) $l s$ quenching, whereas the present mechanism is denoted as the position-type ( $p$-type) $l s$ quenching. It seems that there are unlikely examples of the $s$-type $l s$ quenching in exotic medium mass or heavy nuclei accessible by present and near-future rare-isotope (RI)-beam facilities, whereas there are many $p$-type cases. This means that, in those nuclei, the surface moves out but its slope does not change much.

We investigate the origin of the change of $1 f_{5 / 2}-1 f_{7 / 2}$ splitting in going from ${ }^{68} \mathrm{Ni}$ to ${ }^{78} \mathrm{Ni}$. Note that $1 g_{9 / 2}$ is occupied by more neutrons in this part of the isotopic chain. The change is decomposed according to the components of the Hamiltonian: LS, tensor, and the rest parts. The rest part is comprised of kinetic terms and central interactions including the density-dependent one, and is denoted as Kin + Cent. Figure 3 shows this decomposition for GT2, D1S, and Skyrme SLy4 interactions, demonstrating very clearly the relative importance of those components. We mention that the Kin + Cent and LS parts yield quite similar results among these three interactions. The

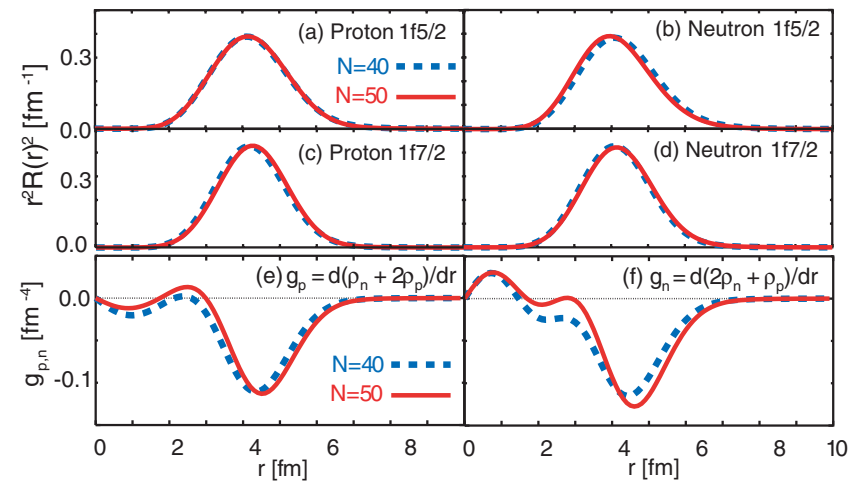

FIG. 2 (color online). (a),(b),(c),(d) $r^{2} R(r)^{2}$ for $1 f_{5 / 2}$ or $1 f_{7 / 2}$. (e),(f) $g_{p}$ and $g_{n}$ [See Eq. (2)]. (a),(c),(e) are for protons, while (b),(d),(f) for neutrons. D1S interaction is used.
Kin + Cent and LS cancel to a large extent for proton splitting. In addition the tensor contribution is largest for both proton and neutron splittings. The LS effect is not much stronger than the Kin + Cent one.

The $Z=28$ gap becomes rather small at ${ }^{78} \mathrm{Ni}$, and the sequence of the $1 f_{5 / 2}$ and $2 p_{3 / 2}$ is reversed between ${ }^{68} \mathrm{Ni}$ and ${ }^{78} \mathrm{Ni}$. This is only a prediction presently, but this trend is consistent with data on $\mathrm{Cu}$ isotopes [19]. Thus, the $Z=$ 28 and $N=28$ magic structures are weakened or disappear in exotic $\mathrm{Ni}$ isotopes. This is also consistent with unusually low-lying $2^{+}$levels [20]. Note that the $N=28$ case is for rather deep hole states and may not be so relevant to lowlying states. The binding energy of ${ }^{68} \mathrm{Ni}$ is almost the same between GT2 and D1S calculations. Because of the tensor force, for ${ }^{78} \mathrm{Ni}$, the GT2 value is smaller by $\sim 5 \mathrm{MeV}$ than the D1S. Note that this argument does not make much sense if ${ }^{78} \mathrm{Ni}$ is deformed.

One may find another interesting feature in the GT2 result: the $Z=N=40$ gap is small for $N \sim 38$, because the neutron $1 f_{5 / 2}$ pulls down $1 g_{9 / 2}$. Deformed low-lying intruder states can become particularly visible in these nuclei. We also note that the $1 g_{9 / 2}$ orbit becomes even lower, if there are less protons in $1 f_{7 / 2}$ as is the case for $\mathrm{Cr}$ compared to $\mathrm{Ni}$. Thus, deformed intruders can be unusually low in some $\mathrm{Cr}$ isotopes.

Figure 4 shows the evolution of the difference between proton $1 h_{11 / 2}$ and $1 g_{7 / 2}$ SPE's as a function of $N$, relative to the corresponding value at $N=64$. It was reported experimentally [21] that proton $1 h_{11 / 2}$ and $1 g_{7 / 2}$ orbits start to get apart at $N=64$, whereas they are rather close to each other up to $N=64$. In Fig. 4 , the change of SPE difference is shown for D1S and GT2 as well as for experiment [21]. The HF calculations are made for the (sub-)shell closures with the neutron numbers shown in Fig. 4. Neutron $3 s_{1 / 2}, 2 d_{3 / 2}$, and $1 h_{11 / 2}$ orbits are assumed to form a common subshell, because they are very close in energy and the pairing correlation should mix them in actual nuclei. As is well known, the mean-field calculations cannot reproduce experimental SPE's too accurately. The GT2 inherits this feature. However, the tensor and

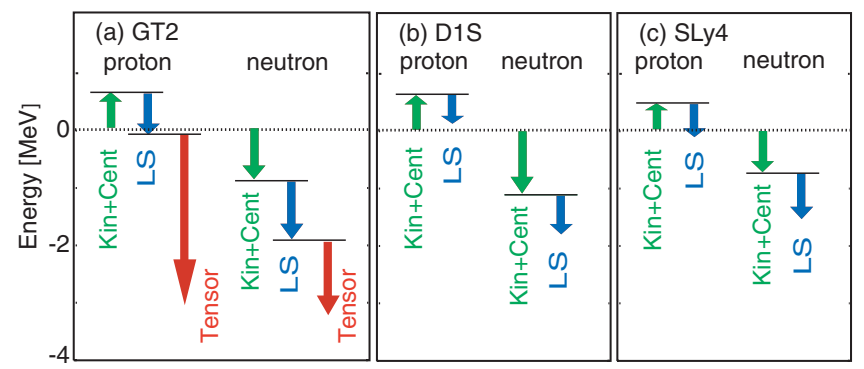

FIG. 3 (color online). Changes of $1 f_{7 / 2}-1 f_{5 / 2}$ splitting from $N=40$ to 50 for (a) GT2, (b) D1S, and (c) SLy4 interactions with decompositions into Kinetic + Central, LS, and tensor parts. 


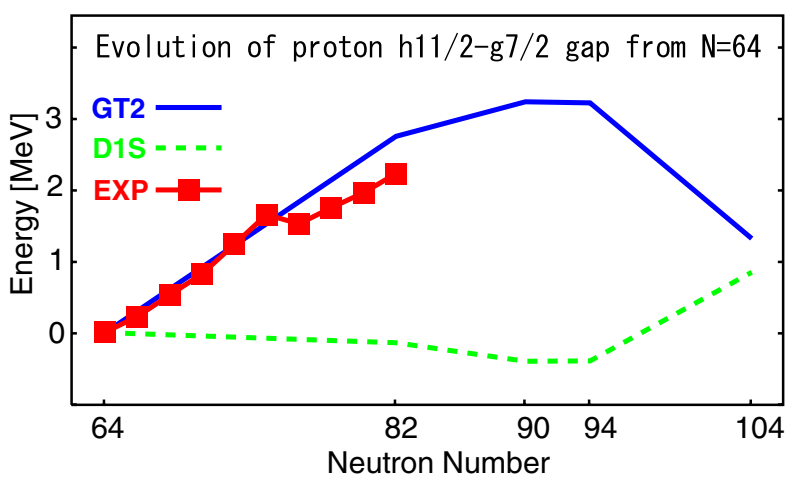

FIG. 4 (color online). Evolution of $1 h_{11 / 2}-1 g_{7 / 2}$ energy gap. The difference from the value of $N=64$ is plotted for experimental data [21] and calculated results with GT2 and D1S interactions.

$l s$-quenching effects are so characteristic that we can see them by taking differences (between levels) and evolutions (as a function of $N$ or $Z$ ). This is the motivation of Fig. 4.

The D1S results do not follow the experimental trend, but show an increase beyond $N=94$, due to the neutronskin effect discussed by using Fig. 2. The GT2 result increases up to $N=90$, and stays constant. It then decreases from $N=94$. This is a tensor-force effect due to the occupancy of $1 h_{9 / 2}$. In fact, the tensor contributions from the occupancy of $1 h_{11 / 2}$ and that of $1 h_{9 / 2}$ are opposite [1]. However, this decrease is canceled partly by the neutron-skin effect as in the D1S result mentioned. The neutron-skin effect here is also of the $p$ type.

Many other mean-field calculations, e.g., Skyrme SLy4, SIII, show results similar to D1S. In fact, a mean potential of a Woods-Saxon-like shape cannot separate $1 h_{11 / 2}$ and $1 g_{7 / 2}$ so much in such a particular way. Thus, the evolution of SPE's over a long chain of isotopes should provide very intriguing cases to be tested experimentally by future RIbeam facilities.

In summary, the evolutions of the nuclear shells are presented in terms of the new mean-field model where the tensor force is implemented explicitly.

The tensor-force effects are of the same order of magnitude as the changes of spin-orbit splitting due to the neutron skin, and thereby both have to be considered simultaneously. Even if the neutron-skin effect is absent or very small, notable tensor effects on SPE's are suggested for examples such as exotic $N=20$ isotones with $Z \sim 10$, exotic Ni isotopes with $N=40-50$, and a long isotopic chain of Sb. Note that many of interesting physics cases are found well inside the drip line, but quite far away from the $\beta$-stability line. The predictions seem to provide us with a paradigm to be tested and developed by future RI-beam physics. We stress that changes in SPE's affect essentially all features of the nuclear structure, e.g., deformation, and also that many of the nuclei involved participate to the astrophysical $r$ process most likely. We point out that a large fraction of the tensor force comes from one $\pi$ exchange, and the present GT2 model includes this mechanism. So, the question is "Are pions so visible in exotic nuclei?"'

The $l s$ quenching has two types. The scale type has been expected [10] but does not appear in nuclei studied in this Letter. The situation could be different if the nucleus sits on or very near the drip line [9]. The position type, which has not been reported in publications, can be found in many cases.

This work was supported in part by a Grant-in-Aid for Specially Promoted Research (No. 13002001) from the MEXT and by the JSPS Core-to-Core Program. This work has been a part of the RIKEN-CNS joint research project on large-scale nuclear-structure calculations. The authors acknowledge Professor A. Gelberg for generous help.

[1] T. Otsuka, T. Suzuki, R. Fujimoto, H. Grawe, and Y. Akaishi, Phys. Rev. Lett. 95, 232502 (2005).

[2] T. H. R. Skyrme, Nucl. Phys. 9, 615 (1959).

[3] D. Vautherin and D. M. Brink, Phys. Rev. C 5, 626 (1972).

[4] J. Decharge, M. Girod, and D. Gogny, Phys. Lett. B 55, 361 (1975).

[5] Fl. Stancu, D. M. Brink, and H. Flocard, Phys. Lett. B 68, 108 (1977).

[6] J. Decharge and D. Gogny, Phys. Rev. C 21, 1568 (1980).

[7] J. F. Berger, M. Girod, and D. Gogny, Nucl. Phys. A428, 23 (1984); Comput. Phys. Commun. 63, 365 (1991).

[8] N. Fukunishi, T. Otsuka, and I. Tanihata, Phys. Rev. C 48, 1648 (1993).

[9] J. Dobaczewski et al., Phys. Rev. Lett. 72, 981 (1994).

[10] RIA Physics White Paper, http://www.phy.anl.gov/ria/riawhitepaper-2000.pdf

[11] B. S. Pudliner et al., Phys. Rev. C 56, 1720 (1997).

[12] G. Bertsch, J. Borysowicz, H. McManus, and W. G. Love, Nucl. Phys. A284, 399 (1977).

[13] T. Otsuka et al., Phys. Rev. Lett. 87, 082502 (2001).

[14] E. Becheva et al., Phys. Rev. Lett. 96, 012501 (2006).

[15] M. Belleguic et al., Phys. Rev. C 72, 054316 (2005).

[16] Y. Utsuno et al., Phys. Rev. C 70, 044307 (2004).

[17] V. Tripathi et al., Phys. Rev. Lett. 94, 162501 (2005).

[18] G. Neyens et al., Phys. Rev. Lett. 94, 022501 (2005).

[19] H. Grawe et al., Eur. Phys. J. A 25, 357 (2005).

[20] M. Sawicka et al., Eur. Phys. J. A 20, 109 (2003).

[21] J. P. Schiffer et al., Phys. Rev. Lett. 92, 162501 (2004). 